\title{
Anorexia Nervosa with Vomiting Episodes: Dermatological and Oral Complications
}

\author{
Elzbieta Paszynska1 Agata Dutkiewicz ${ }^{2}$ Agata Osinska ${ }^{2}$ Marta Mozol-Jursza ${ }^{2}$ Natalia Smalc ${ }^{2}$ \\ Marta Tyszkiewicz-Nwafor ${ }^{2} \quad$ Monika Dmitrzak-Weglarz $^{3} \quad$ Agnieszka Slopien $^{2}$ Dorota Jenerowicz ${ }^{4}$
}

\author{
1Department of Integrated Dentistry, Poznan University of Medical \\ Sciences, Poznań, Poland \\ 2Department of Child and Adolescent Psychiatry, Poznan University \\ of Medical Sciences, Poznań, Poland \\ ${ }^{3}$ Department of Psychiatry, Psychiatric Genetics Unit, Poznan \\ University of Medical Sciences, Poznań, Poland \\ ${ }^{4}$ Department and Clinic of Dermatology, Poznan University of \\ Medical Sciences, Poznań, Poland
}

Address for correspondence Elzbieta Paszynska, DDS, MSc, PhD, Department of Integrated Dentistry, Poznan University of Medical Sciences, Bukowska street 70, Poznan, 60-812, Poland (e-mail: paszynska@ump.edu.pl).

\begin{abstract}
Keywords

- anorexia nervosa

- vomiting

- xerosis

- dental erosion
\end{abstract}

\section{Introduction}

In the group of patients with anorexia nervosa (AN), regular vomiting is noted less frequently; based on literature data, it can be estimated that about half of the patients are diagnosed with bulimic AN. ${ }^{1-3}$ Due to this fact, we find little information on changes in the skin and oral cavity caused by vomiting in AN patients. ${ }^{4}$

The aim of the study was to present three female patients diagnosed with AN with significant skin and oral lesion disturbances after vomiting/purging episodes.

\section{Case Descriptions}

\section{Psychiatric Interview and Assessment of Mental State} Case 1

F.G. was admitted to the Clinic of Child and Adolescent Psychiatry at the age of 17 years. As per the standard guidelines, any detail that reveals patient's identity were not included. At the time of admission, she weighed $35.5 \mathrm{~kg}$ with a height of $158 \mathrm{~cm}$ (body mass index $[\mathrm{BMI}]=14.2 \mathrm{~kg} / \mathrm{m}^{2}$ ). This was the first psychiatric hospitalization in the patient's life. The patient was properly oriented in multiple directions. Her mood was depressed, psychomotor drive normal, and affect appropriate. She denied psychotic symptoms, any thoughts, or intentions of suicide.

F.G. started slimming a year before hospitalization. About 3 to 4 months later, her monthly bleeding stopped'. Half a year before hospitalization in the Clinic, because of cachexia, she was hospitalized in the Pediatric Department. After discharge, she began psychiatric treatment and psychotherapy. There were hunger pains and periodic vomiting. The patient reported that vomiting was a compensatory action of binge eating and claimed that it was a spontaneous effect of stress. The weight of the patient increased to $44 \mathrm{~kg}$. This increased
DOI https://doi.org/ 10.1055/s-0040-1705073 ISSN 1305-7456.
License terms

()(1) $\Theta \circledast$ 
weight made her decide to lose weight again and weight loss was such that hospitalization was necessary.

In the first week of hospitalization, the patient was very reluctant to eat; sometimes hiding meals and limiting the served portion in different ways. In the second week of hospitalization, there was abdominal pain and constipation; she was consulted surgically, laxatives were applied, and the pain subsided. From the beginning, GFR was maintained at 42 to $46 \mathrm{~mL} / \mathrm{min} / 1.73 \mathrm{~m}^{2}$. The patient was also consulted by a nephrologist who diagnosed a reduced peritoneal dialysis requiring further nephrological control, but currently does not require renal replacement therapy. Due to the reduced mood, mianserin was added at $10 \mathrm{mg}$ daily in one evening dose. The patient was also treated dermatologically due to facial skin conditions associated with atopic dermatitis.

Gradually, the patient became more and more cooperative, began to participate in group therapy and her weight began to increase. The parents refused to participate in family therapy.

At the time of submitting the article for print, the patient was still hospitalized with a weight of $41.5 \mathrm{~kg}$. The patient's mood improved, more active, more motivated to treat herself, and stated that she is less concerned with her appearance and weight and has an improved relationship with her parents. Stomach aches and constipation did not occur, but a lowered GFR was still evident and her skin condition improved.

\section{Case 2}

Patient K.L., aged 14, was referred to the Department of Child and Adolescent Psychiatry from another psychiatric hospital, where she was hospitalized and diagnosed with AN, bulimic, for a month. As per the standard guidelines, any detail that reveals patient's identity were not included. At the time of admission, she weighed $39 \mathrm{~kg}$ at $161.5 \mathrm{~cm}\left(\mathrm{BMI}=14.9 \mathrm{~kg} / \mathrm{m}^{2}\right)$. The patient was well oriented, with decreased mood and activity, appropriate affect, lack of psychotic symptoms, and suicidal thoughts or intentions.

According to the report of the girl and her parents, she had been eating limited meals for a year, undertaking additional physical activity, and provoking vomiting after meals. Half a year later, her menstrual cycle was halted, mood reduced, apathy set in, and evident withdrawal from social contacts could be observed. There was a growing concern in relation to food intake and the fear of gaining weight. The patient admitted to daily vomiting, but did not accept the need for treatment.

During hospitalization, the girl took meals with a distinct reluctance, felt increasing anxiety after consumption, and had difficulty in accepting a gradual increase in body weight. She denied vomiting during her stay in the clinic. Pharmacotherapy was used: sertraline up to $50 \mathrm{mg} /$ day, olanzapine up to $5 \mathrm{mg} /$ day, and the patient refused to participate in group therapy. She was involved in family therapy with her parents and was advised to follow this up after discharge. After 3 months of hospitalization, there was an improvement in mood and psychomotor drive, weight rising to $43.9 \mathrm{~kg}$ $\left(\mathrm{BMI}=16.6 \mathrm{~kg} / \mathrm{m}^{2}\right)$.

\section{Case 3}

Diagnosis of AN (bulimic type) in a third patient, 13-yearold girl B.B., was diagnosed during an additional psychiatric consultation in the Department of Endocrinology. She was hospitalized due to her menstrual cycle stopping and reported losing $15 \mathrm{~kg}$ body weight in a year. As per the standard guidelines, any detail that reveals patient's identity were not included. At the beginning of the first hospitalization in the Department of Child and Adolescent Psychiatry, the patient weighed $37.5 \mathrm{~kg}$ at $166 \mathrm{~cm}\left(\mathrm{BMI}=13.4 \mathrm{~kg} / \mathrm{m}^{2}\right)$ and was properly oriented in several directions. A low mood and reduced psychomotor drive was observed, and the affect was diagnosed appropriate. Further, the girl reported suicidal thoughts.

The patient confessed to binge eating, showed a disturbed image of her body and fear of being overweight. The appearance of the symptoms occurred during the intensified conflict between the girl's parents. During the hospitalization, she consumed food with apparent reluctance and also complained of gastrointestinal discomfort-heartburn and reflux of food into the esophagus, which she used to provoke by vomiting after meals. Gastroscopy was performed which showed chronic gastric and duodenal inflammatory lesions without erosions in the esophagus. A traumatic test with negative result was also performed. Further follow-up observation was advised into gastrointestinal reflux, including pharmacotherapy-omeprazole $40 \mathrm{mg} /$ day, probiotic and vitamin C, D, K, chlorchinaldol.

During 3 months of hospitalization, the patient received sertraline up to $75 \mathrm{mg} /$ day and mirtazapine up to $15 \mathrm{mg} /$ day. She participated in an individual psychological interview, and group and family therapy sessions were also conducted. Weight gain $\left(\mathrm{BMI}=16.0 \mathrm{~kg} / \mathrm{m}^{2}\right)$ and appropriate psychomotor drive was observed, but there were still signs of depression and low self-esteem present. It was recommended to follow psychotherapy and outpatient treatment.

After 3 months, the patient was readmitted to the clinic in an acute mode after posting a note of suicidal resignation on one of the social networking sites. During the psychiatric examination, the girl denied any thoughts and intentions of suicide. Significant underweight $\left(B M I=15.1 \mathrm{~kg} / \mathrm{m}^{2}\right)$ and laxative behavior persisted. During hospitalization, the patient blatantly refused to participate in therapeutic activities and did not establish relations with other patients. She was withdrawn from care in a condition that allows for ambulatory treatment.

Less than half a year later, the patient was hospitalized in another psychiatric hospital due to progressive weight loss. Because of the slight increase in weight $(0.3 \mathrm{~kg}$ per month) she was transferred again to the Child and Adolescent Psychiatry Clinic $\left(B M I=14.3 \mathrm{~kg} / \mathrm{m}^{2}\right)$. The patient was also revealed to have a depressed mood and thoughts of suicidal resignation.

During initial hospitalization, B.B. showed low motivation for treatment was apathetic, with a tendency for social isolation. She admitted to provoking vomiting during her hospital stay, but stressed that it was related to the reflux 
of food in the course of gastroesophagus reflux. Pharmacotherapy was continued and participation in group therapy as well as psychoeducation focused on nutrition has been proposed. The patient agreed to these therapeutic activities, over time exhibited increasing commitment, and motivation to treatment, also declared ceasing to provoke vomiting. Dietary counseling was provided, which provided nutritional advice to relieve the symptoms of reflux. The girl at a relatively slow pace gained weight, after 3 months of hospitalization received a BMI $=16.0 \mathrm{~kg} / \mathrm{m}^{2}$. The mood and drive of the patient improved; she did not declare any thoughts and intentions of suicide. Asked for further normalization of body weight and treatment of AN, there was a great deal of anxiety, pointing to the multitude of secondary benefits of the disease.

\section{Results of Oral and Dermatological Examinations}

All three girls had similar hygienic habits: brushed their teeth twice a day and did not regularly use additional hygienic or preventive procedures at home or in the dentist's office. They did not report any dental hypersensitivity during brushing and eating. Only patient B.B. was aware of the loss of hard tissue that occurred in the mouth due to vomiting.

In patient F.G., a slight tooth surface loss was observed, especially on the palate side of the upper teeth, whose location could be attributed to erosive cavities. Due to the long-term neglect of oral hygiene, the surfaces of the teeth were additionally covered with mineralized bacterial plaque, making it difficult to assess these surfaces visually. Similarly, calculus and tartar were located on the lower front teeth

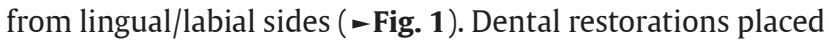
in molars on the occlusal surface were also eroded. These fillings, due to the loss of the original mass, showed surface margins and lack of adhesion, their condition suggesting the need for immediate dental care and replacement. In the oral mucosal examination, the tongue was covered with a white, removable coating. In the projection of the occlusal line of the teeth, keratinization was observed (linea alba).

Dermatologically in the F.G. subject examination, from early childhood, she was found to have suffered from atopic dermatitis-skin ailments are all year round. Home dust mite, pollen, and grass allergy were confirmed. The patient

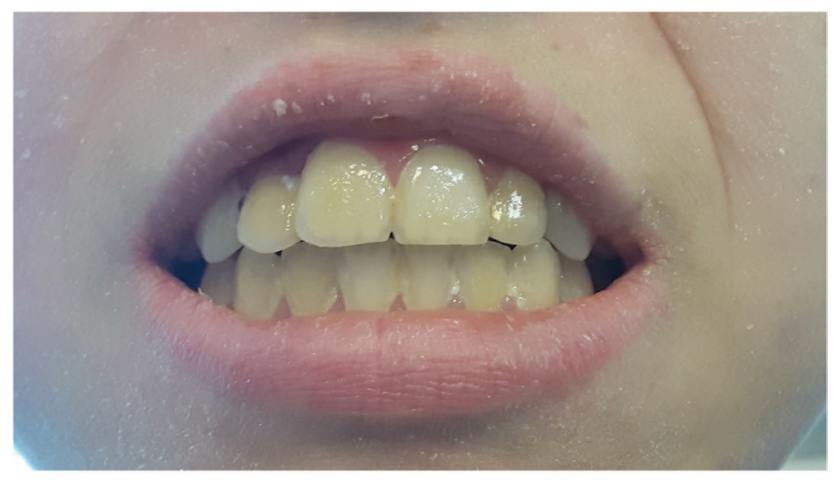

Fig. 1 Erosive (noncarious) enamel loss on the labial surface of upper/lower front teeth in the course of permanent vomiting. Dental plaque and calculus. receives a specific immunotherapy for household dust mite allergens in the classic form (subcutaneous injection).

Generally, substantial dry skin (xerosis) of the whole body, including the scalp hair, was found in the case of the subject. On the face (especially in the area of eye sockets), there was found to be erythema and minor skin peeling ( $\mathbf{- F i g . 2}$ ). She complained about severe skin pruritus. Intensive moisturizing and skin lubrication, pimecrolimus cream, and bilastine $20 \mathrm{mg}$ daily were prescribed. After a week, the clinical condition improved.

In patient K.L. the oral examination showed less tooth surface loss, only within the enamel of the upper front teeth. The surfaces of these teeth were characterized by smoothing, matting, and increased translucency of the incisal edges of the enamel without revealing the dentine. Exfoliative dermatitis was noted, associated with dryness and lip fractures, and atrophic-erythematous lesions were seen in the mucosal surfaces of the cheekbones.

The patient K.L. presented with bordered erythema and edema localized within the skin of distal interphalangeal joints and periungual area as well as both hands. Small erosions and scaling may suggest self-induced trauma caused by picking at the skin. Moreover, redness and skin thickening could be observed over proximal interphalangeal joints of both index fingers, which could be considered as a mildly expressed Russell's sign ( $\boldsymbol{- F i g . 3}$ ). As for other dermatological signs, also cheilitis and angular stomatitis could be observed.

In patient B.B. erosive loss of enamel was in most places complete, as the dentine revealed on a large part of the teeth ( - Fig. 4). In addition, the congenital absence of maxillary second incisors caused wide gaps between incisors, canines, and upper premolars and thus intensified by reduced enamel thickness on both the palatal and interproximal surfaces of these teeth. This observation was not manifested in previous patients. All surfaces of the teeth were smoothed, matted, and characterized by increased enamel translucency so that the front teeth had a much thinner anterior-posterior dimension. Molar teeth had maximal flattened cusps, pits, and fissures on the occlusal

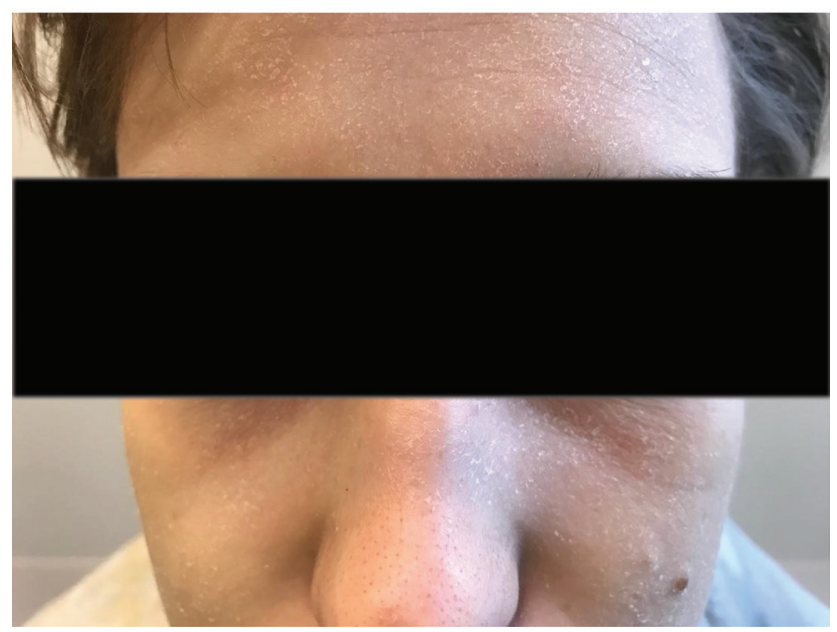

Fig. 2 Extreme xerosis of the face in a patient suffering from anorexia with concomitant atopic dermatitis. 


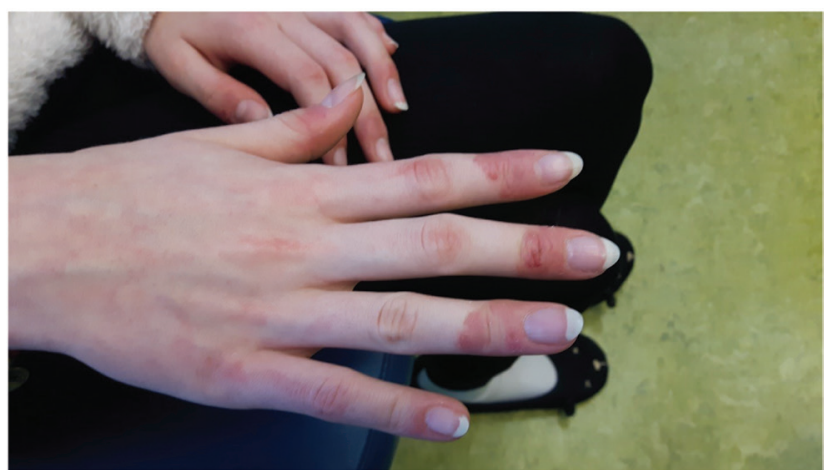

Fig. 3 Sharply bordered erythema localized within the skin of distal interphalangeal joints and periungual area and of both hands together with redness and skin over proximal interphalangeal joints of both index finger (mildly expressed Russell's sign).

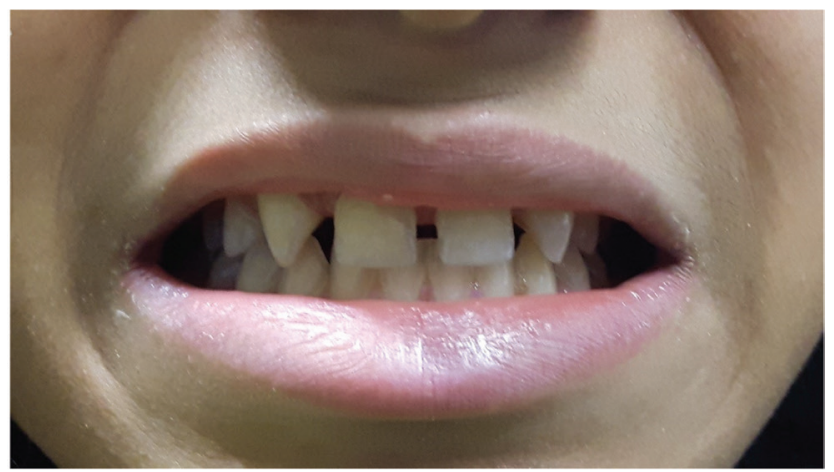

Fig. 4 Erosive (noncarious) enamel loss of structure on the labial and interproximal surfaces of upper/lower front teeth caused by permanent vomiting.

surfaces. In the oral mucosal examination, the tongue was covered with a white and removable coating, while in the projection of the occlusal line of the teeth, keratinization was observed in the mucous membrane of the cheekbones in the form of a ligament (linea alba).

As for other dermatological signs, also xerosis, cheilitis and angular stomatitis could be observed.

\section{Discussion}

Two described patients, K.L. and B.B., have been subjected to several psychiatric hospitalizations, presenting a reduced mood and tendency to limit social relationships. F.G., who reported the lowest frequency of vomiting provocation, showed mood and social functioning not significantly different from normal. This is consistent with the results of clinical trials suggesting more complex psychopathology among patients who are more likely to provoke vomiting to prevent weight gain-above all, greater depressiveness and anxiety compared with patients with restrictive-type disease. ${ }^{2,3}$ As with the diagnosis of bulimia, patients with AN of the bulimic type are attributed to greater mood fluctuation and impulsivity, ${ }^{5,6}$ which translates into undertaking unhealthy behavior-including purging and self-aggression. Pryor et $\mathrm{al}^{7}$ reported more frequent episodes of impulsive theft and suicide attempts or self-aggressive behavior for the above than patients affected from other types of eating disorders. K.L. and B.B. declared their willingness to self-harm in difficult situations. Weight gain during hospitalization was relatively slow and unstable, which was most likely due to the involvement of the girls in laxative behavior. This negatively affected the course of therapeutic activities and extended the hospitalization. Many authors in the literature recognized that the presence of purging behavior in patients with AN could influence an adverse prognosis.,

It is worth noting that in laboratory tests, all patients had adverse changes in blood morphology-characteristic of anemia and abnormalities in the lipid profile (hypertriglyceridemia and over exceeding LDL cholesterol levels). In contrast, no specific water-electrolyte disturbances were observed, characteristic for chronic vomiting. The results in the electrolyte range were normal, which could be mistaken for suggesting minor complications resulting from regular stimulation of gastric vomiting. However, dental and dermatological examinations revealed serious changes in the oral cavity of the hospitalized girls and in the case of F.G. and K.L., additionally dermatological ones.

\section{Study of Oral Manifestations}

Changes in the oral mucosa may have been caused by factors such as pH lowering, mechanical, and chemical irritation of the mucous membrane of the oral cavity; hypersensitivity of the mucous membrane caused by nutritional deficiencies (e.g., vitamins A and B) and the underlying stress associated with primary disease. ${ }^{10,11}$ During the fasting period, the atrophic-erythematous alterations of the oral mucosa can occur with inflammatory conditions on the dorsal surface of the tongue and red lips. ${ }^{12,13}$ Long-lasting chewing of the cheeks give a thickening of the oral mucosa at the level of cheeks known as linea alba.

On the other hand, the presence of removable plaque and white sediment on the dorsal surface of the tongue may be attributed to some patients' hygienic neglect and vomiting. ${ }^{10-13}$

In all presented subjects, the predominant change was the loss of hard tissue due to chemical erosion. Multicentered oral study on more than 200 patients with eating disorders confirms the presence of dental erosion with a frequency of $63 \%{ }^{14,15}$ Occurrence of other clinical changes in the oral cavity, which may suggest a regular incidence of vomiting/ reflux, such as tooth decay and inflammation of the mucosa are estimated at $\pm 30 \% .{ }^{16}$

The primary cause of erosive lesions in AN patients seems to be the return of stomach contents to the oral cavity. ${ }^{17-20}$ Therefore, the loss of enamel occurs in the first phase on the occlusal surfaces of the lateral teeth and palatal surfaces of the upper incisors. The location of erosion is not accidental and is related to the movement of the acid content of the stomach through the oral cavity and retention of its remnants on the dorsal surface of the tongue. In addition to the palatal surfaces of the incisors, the loss of enamel occurs on natural or restored occlusal surfaces of teeth.

Studies in eating disorders have shown that the first signs of loss of hard dental tissue may occur after a half-year 
of vomiting incidences. ${ }^{21-23}$ Milosevic and Dawson ${ }^{21}$ suggest that it is highly probable that over 1,100 episodes of vomiting (3-5 years) will lead to a chemical dissolution of tooth tissue in the form of erosion. The range of erosion changes may also be affected by both high carbohydrate and low carbohydrate diets with acidic pH, as well as high salivary viscosity associated with a decreased salivary flow rate. ${ }^{18,21}$

Therefore, it is advisable that patients in the course of repeated vomiting additionally stimulate salivary secretion and raise $\mathrm{pH}$, providing mineral buffering ingredients to the mouth. According to Otsu et al, drinking still water before and after stimulation of vomiting may prove to be a protective measure against erosion. ${ }^{18}$ Brushing the teeth immediately after vomiting stimulates the structure of the enamel to irreversible loss and is not recommended. ${ }^{20}$

During vomiting episodes in patients with AN simple recommendations such as increased water consumption, regular water rinsing or mouthwash with appropriate fluoride, and hydroxyapatite concentrations, selection of toothpaste for its components, right brushing time, and type of brush should be part of dental care run by a dentist. ${ }^{24-29}$

In the last years, nonfluoride remineralization systems have been developed and commercialized. These systems are based on calcium phosphates such as particulate hydroxyapatite. They do not show potential risks associated with fluorides, but can facilitate erosion/caries control. ${ }^{30}$

\section{Study of Dermatological Status}

Various abnormalities as the most common dermatological findings were xerosis, cheilitis, bodily hypertrichosis, alopecia, dry scalp hair, acral coldness, acrocyanosis, periungual erythema, gingival changes, nail changes, and calluses on the dorsum of the hand. The authors emphasize that there are remarkable similarities between cutaneous manifestations in AN and HIV infection that have to be taken into account by dermatologists and other specialists. Moreover, according to Glorio et al, ${ }^{31}$ patients suffering from AN develop early stereotype skin changes. Principal dermatological features found in 200 patients included: xerosis, acne, alopecia, caries, hypertrichosis, and Russell's sign in bulimia nervosa and xerosis and hypertrichosis, acne, alopecia, and caries in $\mathrm{AN}^{30}$

As for children and adolescents, Schulze et a $\mathrm{l}^{32}$ observed xerosis of the skin, diffuse hypertrichosis, acrocyanosis, scars, diffuse effluvium, artifacts, brittle nails, and onychophagia. Significant association was found between the presence of hypertrichosis and the existence of amenorrhea or a body mass index of less than $16 \mathrm{~kg} / \mathrm{m}^{2}$. It would appear that this age group shows dermatologic features similar to those reported in older patients; however, special findings in this age group include extensive lanugo hair and signs of auto-aggressive behavior. ${ }^{32}$

The most frequent cutaneous manifestations of eating disorders are described in $\boldsymbol{\sim}$ Table. 1 . Based on the analysis of the three subjects diagnosed with AN, the observed erosive loss of hard dental tissues and changes in the macroscopic structure of the oral mucosa can be said to be primarily a result
Table 1 The most frequent ("guiding") cutaneous manifestations of eating disorders

\begin{tabular}{|l|l|}
\hline $\begin{array}{l}\text { Cutaneous manifestations } \\
\text { secondary to starvation } \\
\text { and/or malnutrition }\end{array}$ & $\begin{array}{l}\text { Xerosis, hair and nail fragility, lanu- } \\
\text { go-like body hair, carotenodermia, } \\
\text { acne, acrocyanosis, and pruritus }\end{array}$ \\
\hline $\begin{array}{l}\text { Cutaneous manifesta- } \\
\text { tions due to self-induced } \\
\text { vomiting }\end{array}$ & Russell's sign and perimyolysis \\
\hline $\begin{array}{l}\text { Manifestations secondary } \\
\text { to laxative or diuretic use }\end{array}$ & $\begin{array}{l}\text { Fixed drug eruption, photosensi- } \\
\text { tivity, urticaria, and finger clubbing } \\
\text { (abuse of laxatives with senna) }\end{array}$ \\
\hline $\begin{array}{l}\text { Skin symptoms as a con- } \\
\text { sequence of concomitant } \\
\text { psychiatric morbidity }\end{array}$ & $\begin{array}{l}\text { Self-induced trauma, trichotilloma- } \\
\text { nia, and body image disturbance }\end{array}$ \\
\hline
\end{tabular}

of laxative behavior, which has disturbed the homeostasis of the maxillofacial region. It is believed that in the future the consequences of poor oral health can be difficult to modify as an aesthetic problem.

Undoubtedly, early diagnosis influences prognosis in the course of eating disorders. Since the skin symptoms may be the only detectable evidence of hidden eating disorder, a dermatologist plays an important role in their proper recognition. It may not be an easy task with regard to 40 cutaneous manifestations and a systematically increasing list of reports; however, particular vigilance may be recommended in the case of patients who tend to minimize or deny their disorder.

The cases of AN described above therefore indicate the need for multispecialty medical care, including constant dental and dermatological observation as well as treatment.

\section{Conflict of Interest}

None declared.

\section{References}

1 Peat C, Mitchell JE, Hoek HW, Wonderlich SA. Validity and utility of subtyping anorexia nervosa. Int J Eat Disord 2009;42(7):590-594

2 Casper RC, Eckert ED, Halmi KA, Goldberg SC, Davis JM. Bulimia. Its incidence and clinical importance in patients with anorexia nervosa. Arch Gen Psychiatry 1980;37(9):1030-1035

3 Garner DM, Garner MV, Rosen LW. Anorexia nervosa "restricters" who purge: implications for subtyping anorexia nervosa. Int J Eat Disord 1993;13(2):171-185

4 Clauwaert N, Jones MP, Holvoet L, et al. Associations between gastric sensorimotor function, depression, somatization, and symptom-based subgroups in functional gastroduodenal disorders: are all symptoms equal? Neurogastroenterol Motil 2012;24(12):1088-e565

5 Garfinkel PE, Moldofsky H, Garner DM. The heterogeneity of anorexia nervosa. Bulimia as a distinct subgroup. Arch Gen Psychiatry 1980;37(9):1036-1040

6 Garner DM, Garfinkel PE, O'Shaughnessy M. The validity of the distinction between bulimia with and without anorexia nervosa. Am J Psychiatry 1985;142(5):581-587

7 Pryor T, Wiederman MW, McGilley B. Clinical correlates of anorexia nervosa subtypes. Int JEat Disord 1996;19(4):371-379

8 Halmi K, Brodland G, Loney J. Prognosis in anorexia nervosa. Ann Intern Med 1973;78(6):907-909

9 Deter HC, Herzog W. Anorexia nervosa in a long-term perspective: results of the Heidelberg-Mannheim Study. Psychosom Med 1994;56(1):20-27 
10 Romanos GE, Javed F, Romanos EB, Williams RC. Oro-facial manifestations in patients with eating disorders. Appetite 2012;59(2):499-504

11 Lo Russo L, Campisi G, Di Fede O, Di Liberto C, Panzarella V, Lo Muzio L. Oral manifestations of eating disorders: a critical review. Oral Dis 2008;14(6):479-484

12 Back-Brito GN, da Mota AJ, de Souza Bernardes LÂ, et al. Effects of eating disorders on oral fungal diversity. Oral Surg Oral Med Oral Pathol Oral Radiol 2012;113(4):512-517

13 Paszyńska E, Słopień A, Ślebioda ZS, Dyszkiewicz-Konwińska M, Weglarz M, Rajewski A. Macroscopic evaluation of the oral mucosa and analysis of salivary $\mathrm{pH}$ in patients with anorexia nervosa [article in Polish]. Psychiatr Pol 2014;48(3):453-464

14 Conviser JH, Fisher SD, Mitchell KB. Oral care behavior after purging in a sample of women with bulimia nervosa. J Am Dent Assoc 2014;145(4):352-354

15 Uhlen MM, Tveit AB, Stenhagen KR, Mulic A. Self-induced vomiting and dental erosion-a clinical study. BMC Oral Health 2014;14-92

16 Willumsen T, Graugaard PK. Dental fear, regularity of dental attendance and subjective evaluation of dental erosion in women with eating disorders. Eur J Oral Sci 2005;113(4):297-302

17 Johansson AK, Norring C, Unell L, Johansson A. Eating disorders and oral health: a matched case-control study. Eur J Oral Sci 2012;120(1):61-68

18 Otsu M, Hamura A, Ishikawa Y, Karibe H, Ichijyo T, Yoshinaga Y. Factors affecting the dental erosion severity of patients with eating disorders. Biopsychosoc Med 2014;8:25

19 Hermont AP, Oliveira PA, Martins CC, Paiva SM, Pordeus IA, Auad SM. Tooth erosion and eating disorders: a systematic review and meta-analysis. PLoS One 2014;9(11):e111123

20 Robb ND, Smith BG, Geidrys-Leeper E. The distribution of erosion in the dentitions of patients with eating disorders. $\mathrm{Br}$ Dent J 1995;178(5):171-175

21 Milosevic A, Dawson LJ. Salivary factors in vomiting bulimics with and without pathological tooth wear. Caries Res 1996;30(5):361-366
22 Grippo JO, Simring M, Coleman TA. Abfraction, abrasion, biocorrosion, and the enigma of noncarious cervical lesions: a 20-year perspective. J Esthet Restor Dent 2012;24(1):10-23

23 Altshuler BD. Eating disorder patients. Recognition and intervention. J Dent Hyg 1990;64(3):119-125

24 Fairburn CG. Cognitive Behavior Therapy and Eating Disorders. New York, NY: Guilford Press; 2008

25 Davis R, Olmsted M, Rockert W, Marques T, Dolhanty J. Group psychoeducation for bulimia nervosa with and without additional psychotherapy process sessions. Int J Eat Disord 1997;22(1):25-34

26 Vella-Zarb RA, Mills JS, Westra HA, Carter JC, Keating L. A Randomized controlled trial of motivational interviewing + selfhelp versus psychoeducation + self-help for binge eating. Int Eat Disord 2015;48(3):328-332

27 Aranha AC, Eduardo CdeP, Cordás TA. Eating disorders part II: clinical strategies for dental treatment. J Contemp Dent Pract 2008;9(7):89-96

28 Kisely S, Baghaie H, Lalloo R, Johnson NW. Association between poor oral health and eating disorders: systematic review and meta-analysis. Br J Psychiatry 2015;207(4):299-305

29 Milosevic A. Eating disorders and the dentist. Br Dent J 1999;186(3):109-113

30 Schlagenhauf U, Kunzelmann K-H, Hannig C, et al. Impact of a non-fluoridated microcrystalline hydroxyapatite dentifrice on enamel caries progression in highly caries-susceptible orthodontic patients: a randomized, controlled 6-month trial. J Investig Clin Dent 2019;10(2):e12399

31 Glorio R, Allevato M, De Pablo A, et al. Prevalence of cutaneous manifestations in 200 patients with eating disorders. Int J Dermatol 2000;39(5):348-353

32 Schulze UM, Pettke-Rank CV, Kreienkamp M, et al. Dermatologic findings in anorexia and bulimia nervosa of childhood and adolescence. Pediatr Dermatol 1999;16(2):90-94 